\title{
Early stage squamous cell carcinoma of the tonsil presenting with multiple organ metastases including skin and brain after successful local treatment
}

\author{
Susovan Banerjee ${ }^{1}$, Dipankar Kundu' ${ }^{2}$ Mukti Mukherjee ${ }^{1}$, Pradip Kumar Maiti ${ }^{1}$ \\ ${ }^{I}$ Department of Radiotherapy, NRS Medical College, Kolkata 700014, West Bengal, India. \\ ${ }^{2}$ Department of Surgery, NRS Medical College, Kolkata 700014, West Bengal, India.
}

Correspondence to: Dr. Susovan Banerjee, Department of Radiotherapy, NRS Medical College, Kolkata 700014, West Bengal, India.

E-mail: drsusovan@gmail.com

\section{A B S T R A C T}

Early stage carcinoma of the tonsil is curable, and the incidence of systemic metastasis is very low and central nervous system involvement is very rare. A patient diagnosed with early stage tonsillar carcinoma treated with chemoradiation was followed by brachytherapy boost. One and half years after completion of treatment, the patient presented with disseminated metastasis to the skin, lung, liver, bone, and brain. He had all favorable prognostic parameters except being a young adult.

Key words: Brain metastasis, distant metastasis, early stage cancer tonsil, skin metastasis

\section{Introduction}

Early stage carcinoma of the tonsil is curable with a very low incidence of distant metastasis. Advanced stage carcinoma of the oropharynx has a probability of distant metastasis from $15 \%$ to $20 \%{ }^{\left[{ }^{[1]}\right.}$ Considering all stages and sub-sites of head and neck cancer, the incidence of distant metastasis is reported to be of about $10-15 \%{ }^{[2]}$ Evidence of distant metastasis in early stage disease is not available in the literature. We diagnosed a case of early stage carcinoma tonsil that had been treated effectively with concurrent chemoradiotherapy followed by brachytherapy boost. The patient had locally controlled disease but developed multiple visceral metastases through hematogeneous dissemination within a few months of treatment completion.

\section{Case Report}

A 34-year-old Indian male with a history of being a chronic smoker and alcoholic presented with complaints of dysphagia, foreign body sensation during deglutition of 3 months duration. On physical examination, the patient appeared to be in a good performance and adequate nutritional status. Clinical examination revealed a proliferative growth at left tonsil without evidence of any palpable neck nodes. Direct laryngoscope revealed

\begin{tabular}{|l|l|}
\hline \multicolumn{2}{|c|}{ Access this article online } \\
\hline Quick Response Code: & Website: \\
\hline & www.jcmtjournal.com \\
\cline { 2 - 3 } & \\
\hline
\end{tabular}

proliferative growth of $3 \mathrm{~cm}$ diameter at left tonsil; involving the anterior and posterior pillar and encroaching to the soft palate and lateral pharyngeal wall. The valleculae and epiglottis were free. The histopathological report of the tonsillar fossa biopsy indicated moderately differentiated, infiltrating squamous cell carcinoma. Contrast-enhanced computed tomography scan of the head and neck revealed $3.1 \mathrm{~cm} \times 2.2 \mathrm{~cm}$ sized mass at the left tonsillar fossa. In the neck, there was no evidence of lymph node metastasis. Ultrasonography (USG) of the abdomen and pelvis was normal except for mild fatty changes in the liver. Chest skiagram was normal. Hence, the patient was diagnosed as a case of carcinoma tonsil $\mathrm{cT}_{2} \mathrm{~N}_{0} \mathrm{M}_{0}$ and was planned for radical therapy. He was treated with external beam radiotherapy (EBRT) of $6 \mathrm{MeV}$ photon with conventional bilateral portal plan, 50 Gy in 25 fractions, along with 5 cycles of concurrent chemotherapy with cisplatinum (65 mg). Thereafter, a boost radiation by interstitial high-dose-rate brachytherapy of $20 \mathrm{~Gy} / 5$ fractions to residual tumor and tonsillar fossa was given.

After completion of treatment, the patient was asked to visit the follow-up (FU) for clinical evaluation every 3 months. The 1st year of FU was unremarkable. At the FU visit at 18 months, he presented with a recent history of dry cough for more than 1 week. Chest skiagram showed only pneumonitic changes at the lung bases. He was managed conservatively and responded well. At the FU of 20 months, the patient was suffering from backache which has progressed over a short period in spite of taking analgesics. Digital X-ray of the lumbar sacral (LS) spine revealed no obvious findings. However, within a few weeks, the pain increased severely, and the patient was unable to walk or stand without support. Clinically he had a severe tenderness over the lumbar 


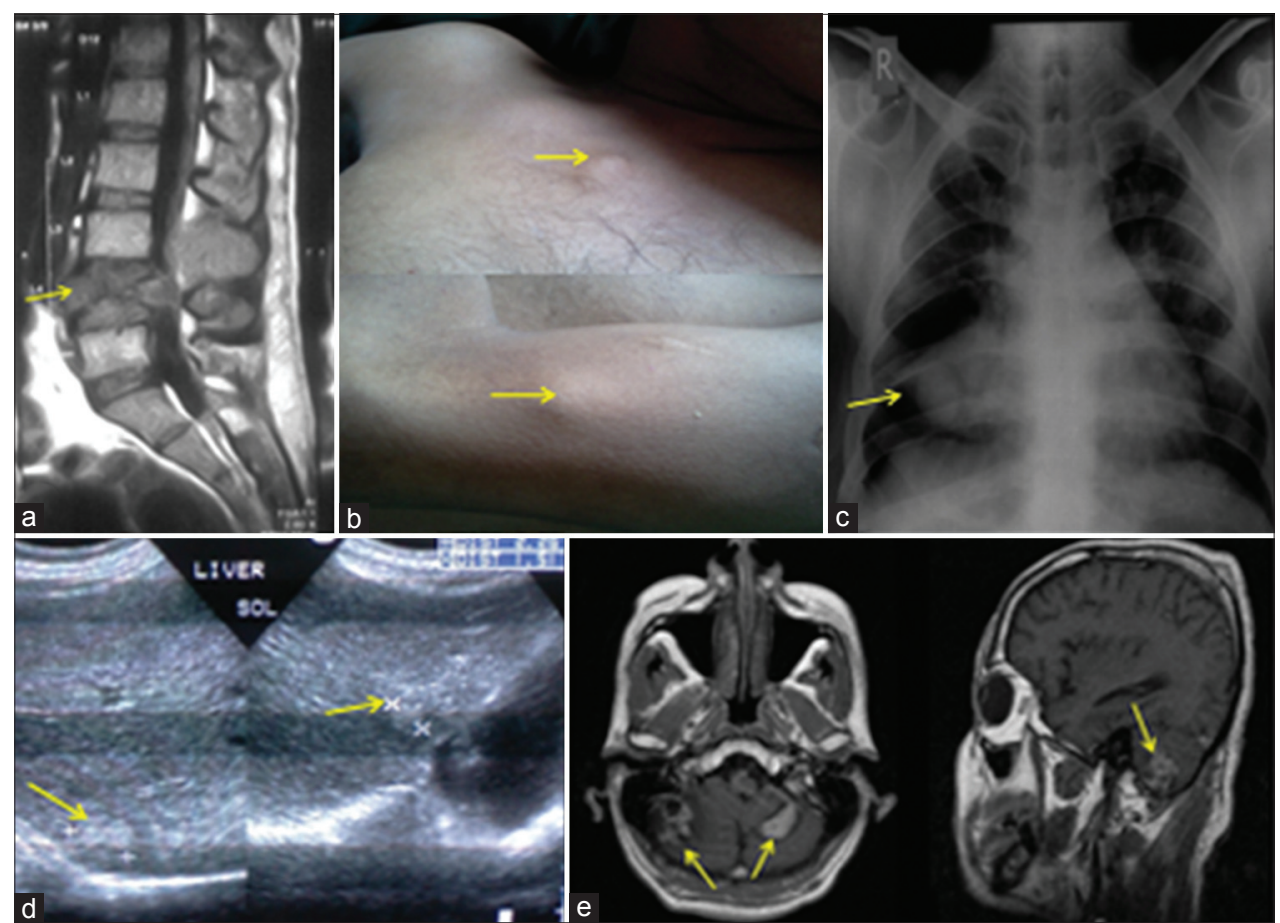

Figure 1: Multiple metastatic sites in a treated case of early stage cancer of tonsil: (a) spine; (b) skin nodules; (c) lungs; (d) liver; (e) brain. The yellow arrows in the respective sites point to the metastatic sites

spine. Magnetic resonance imaging of LS spine showed wedge compression fracture of L4 vertebral body and altered marrow signal intensity with associated periosseous component at L3 and L4 level; it's likely to be a metastasis [Figure 1a].

There was no evidence of loco-regional failure. During clinical examination of the patient multiple subcutaneous nodules were found, mainly in the upper part of the body (over chest, arms, nape of the neck, scalp) [Figure 1b]. Excision biopsy from the nodule over left arm showed histopathological features of metastatic deposit from squamous cell carcinoma. He underwent a metastatic workup. His chest skiagram showed bilateral, multiple nodular opacities suggestive of metastasis [Figure 1c]. USG whole abdomen also revealed multiple hypoechoic space occupying lesion in both lobes of the liver (largest being $2.25 \mathrm{~cm} \times 1.5 \mathrm{~cm}$ ) [Figure 1d].

Magnetic resonance imaging of brain central nervous system (CNS) showed multiple altered signal intensity in both cerebellar hemispheres and also similar deposits at right parietal, left temporal, parietal and occipital bony calvarium, and adjacent scalp resulting in bony destruction [Figure 1e]. He was treated by palliative radiotherapy (30 Gy in 10 fractions) to whole brain and to lumbar the spine along with other supportive care. He had $50-60 \%$ of pain relief and marginal improvement of neurological symptoms. After treatment, he was taken home and offered best supportive care; he died after 3 months of completion of radiotherapy.

\section{Discussion}

For head and neck squamous cell cancers, only 5\% of cases with loco-regionally controlled disease may present with distant metastasis. ${ }^{[2]}$ Early stage cancer of head and neck is considered to have a good prognosis in general irrespective of sub-site or stage of the disease. Carcinoma tonsil with $\mathrm{T}_{2}$ disease is considered to have $80 \%$ curability. Treatment with EBRT followed by brachytherapy boost is a standard practice. ${ }^{[3]}$ The case merits discussion for a number of reasons. In the first place, this is probably the first case reported in English literature with a head neck malignancy having such widespread metastasis involving lung, liver, bones, skin, and brain. The literature supports that the distant metastasis from carcinoma tonsil commonly spreads to lungs and rarely to bones or liver. ${ }^{[4,5]}$ However, involvement of so many organs is unknown. Second, there has been a very few reports of head and neck cancers metastasizing CNS by the hematogenous route. ${ }^{[1,6]}$ The natural history of the disease in this patient confirms surely that he had a hematogenous metastasis to the CNS along with other organs.

The current treatment options limit the survival of metastatic head and neck cancers patients to $<1$ year ${ }^{[7]}$ and our patient with extensive multiorgan metastasis survived for only 3 months.

We attempted to identify the risk factors for distant metastasis in head and neck carcinoma by retrospective analyses. ${ }^{[4,8]}$ In univariate and multivariate analysis, the most significant factors were neck node 
involvement (number, level of neck node involved) and site of the primary tumor $(P<0.001)$. Among other factors, $\mathrm{T}$ stage of the primary tumor, histopathological grade of tumor, response to treatment, and young age are also mentioned but with varying significance in different studies. In this context, this case had features of favorable prognostic group (stage $\mathrm{cT}_{2} \mathrm{~N}_{0} \mathrm{M}_{0}$, tonsil, moderately differentiated squamous cell carcinoma, excellent loco-regional control) but except for young age. Similar cases need to be reported and documented so that more aggressive FU can be advised in this group of patients.

The reasons for this unusual presentation of such widespread metastasis in a patient with apparently good prognostic factors is not known. However, we came across two very interesting reports while preparing this case report. In a study on prostate cancer patients treated by brachytherapy, the authors concern that the cells liberated at the time of brachytherapy increases the risk of metastatic deposits and may results in a systemic failure, as measured by serum prostate-specific antigen levels. ${ }^{[9]}$ Similar observation had been made in case of a glioblastoma multiforme is treated by brachytherapy. ${ }^{[10]}$ There are no further evidences in this regards, but those interesting incidences need to be reviewed in the context of our case to find out the rarest possibility of any such mechanism.

\section{References}

1. Dobelbower MC, Nabell L, Markert J, Carroll W, Said-Al-Naief N, Meredith R. Cancer of the tonsil presenting as central nervous system metastasis: A case report. Head Neck 2009;31:127-30.
2. León X, Quer M, Orús C, del Prado Venegas M, López M. Distant metastases in head and neck cancer patients who achieved loco-regional control. Head Neck 2000;22:680-6.

3. Chen J, Pappas L, Moeller JH, Rankin J, Sharma PK, Bentz BG, Fang LC, Hayes JK, Shrieve DC, Hitchcock YJ. Treatment of oropharyngeal squamous cell carcinoma with external beam radiation combined with interstitial brachytherapy. Head Neck 2007;29:362-9.

4. Ferlito A, Shaha AR, Silver CE, Rinaldo A, Mondin V. Incidence and sites of distant metastases from head and neck cancer. ORL J Otorhinolaryngol Relat Spec 2001;63:202-7.

5. Goodwin WJ. Distant metastases from oropharyngeal cancer. ORL J Otorhinolaryngol Relat Spec 2001;63:222-3.

6. Djalilian HR, Tekin M, Hall WA, Adams GL. Metastatic head and neck squamous cell carcinoma to the brain. Auris Nasus Larynx 2002;29:47-54.

7. Price KA, Cohen EE. Current treatment options for metastatic head and neck cancer. Curr Treat Options Oncol 2012;13:35-46.

8. Garavello W, Ciardo A, Spreafico R, Gaini RM. Risk factors for distant metastases in head and neck squamous cell carcinoma. Arch Otolaryngol Head Neck Surg 2006;132:762-6.

9. Siddiqua A, Chendil D, Rowland R, Meigooni AS, Kudrimoti M, Mohiuddin M, Ahmed MM. Increased expression of PSA mRNA during brachytherapy in peripheral blood of patients with prostate cancer. Urology 2002;60:270-5.

10. Houston SC, Crocker IR, Brat DJ, Olson JJ. Extraneural metastatic glioblastoma after interstitial brachytherapy. Int J Radiat Oncol Biol Phys 2000;48:831-6.

How to cite this article: Banerjee S, Kundu D, Mukherjee M, Maiti PK.
Early stage squamous cell carcinoma of the tonsil presenting with
multiple organ metastases including skin and brain after successful
local treatment. J Cancer Metastasis Treat $2015 ; 1: 31-3$.
Received: $08-08-2014$; Accepted: $15-11-2014$.
Source of Support: Nil, Conflict of Interest: None declared.

